\title{
Safety and efficacy of Dai-kenchu-to in postoperative infants with gastroschisis ${ }^{*}$
}

\author{
Kohei Otake $^{1 \#}$, Keiichi Uchida ${ }^{1}$, Yuhki Koike ${ }^{1}$, Mikihiro Inoue ${ }^{1}$, Kohei Matsushita ${ }^{2}$, \\ Kiyoshi Hashimoto $^{1}$, Shozo Ide ${ }^{1}$, Yuka Nagano ${ }^{1}$, Masato Kusunoki ${ }^{1 \#}$ \\ ${ }^{1}$ Department of Gastrointestinal and Paediatric Surgery, Mie University Graduate School of Medicine, Mie, Japan \\ ${ }^{2}$ Department of Pediatric Surgery, National Mie Hospital, Mie, Japan

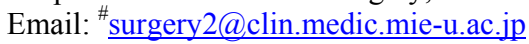

Received 14 August 2012; revised 17 September 2012; accepted 5 October 2012

\begin{abstract}
Purpose: Intestinal dysfunction is a major problem after gastroschisis repair, and causes poor oral intake and poor body weight gain in infants with gastroschisis. Dai-kenchu-to (DKT) is often used in the treatment of gastrointestinal dysfunction in Japan. The purpose of this study was to analyze the effect of DKT on infants with uncomplicated gastroschisis. Methods: In this retrospective cohort study from 1991 to 2009, we assessed 16 infants with uncomplicated gastroschisis. Eight infants received $0.3 \mathrm{~g} / \mathrm{kg} /$ day of DKT for 12 months (group 1) and 8 infants did not receive DKT (group 2). We analyzed their body weight as an indicator of the effect of DKT. Results: There were no significant differences in the background characteristics. The body weight of group 1 was significantly increased at the ages of 9 months $(p=0.0348)$ and 12 months ( $p=0.0097$ ), compared with group 2 . Multivariate analyses revealed that only DKT administration had a significant impact on body weight gain at the ages of 9 months $(p=0.0201)$ and 12 months $(p=$ 0.0111). No side effects related to DKT were reported. Conclusion: DKT administration might worksafely and effectively for improvingthe body weight gain in infants with uncomplicated gastroschisis.
\end{abstract}

Keywords: Gastroschisis; Dai-Kenchu-to; Intestinal Dysfunction; Body Weight Gain

\section{INTRODUCTION}

Gastroschisis is associated with abnormal intestinal motility and nutrient absorption, both of which gradually improve over time in most patients [1]. The introduction of enteral feeding is often delayed for weeks while awaiting the return of bowel function. Gastrointestinal

*Declaration of interest: The authors report no conflicts of interest.

\#Corresponding authors. dysmotility is often treated with prokinetic agents in gastroschisis patients. However, there is little documentation in the literature to support their use. Commonly utilized prokinetic agents include erythromycin, metoclopramide, domperidone and cisapride [2]. A randomized controlled trial of erythromycin versus placebo showed that enterally administered erythromycin did not improve the time required to achieve full enteral feeding compared with placebo [3]. Meanwhile, a similar randomized trial examining the use of cisapride in postoperative neonates, most of whom had gastroschisis, did show a beneficial effect [4]. However, cisapride is only available on a compassionate basis in North America because of its relatively high risk of cardiovascular side effects, and therefore can only be used for severe intestinal dysmotility in infants who have not responded to other agents and who have undergone cardiac evaluation and clearance.

Dai-kenchu-to (DKT) is widely prescribed for patients with gastrointestinal dysfunction, such as postoperative ileus, postoperative intestinal paralysis and adhesive bowel obstructions, by a large number of surgeons at medical institutions, including all university-affiliated hospitals in Japan [5-7]. Several double-blind placebo-controlled studies on DKT are now in progress in Japan and the United States, and the results of a recent clinical pharmacological study clearly indicated that DKT accelerates intestinal transit in healthy humans [8]. The potential beneficial effects of DKT $(0.3 \mathrm{~g} / \mathrm{kg} / \mathrm{day})$ for internal disorders, including irritable bowel syndrome and functional constipation, have also been investigated in pediatric patients [9].

DKT is the most frequently prescribed traditional medicine in Japan [7]. DKT has been in the Japanese market for several decades with proven effectiveness for gastrointestinal hypomotility, including ileus following abdominal surgery, and has very few side effects [6]. DKT extract powder is manufactured through an aqueous extract containing $2.2 \%$ Japanese pepper, $5.6 \%$ processed ginger, $3.3 \%$ ginseng and $88.9 \%$ maltose syrup 
powder, and the main ingredients of DKT include hydroxy- $\alpha$-sanshool (from Japanese pepper), 6-shogaol (from processed ginger) and ginsenoside Rb1 (from ginseng) [7].

Previous pharmacological studies have suggested that DKT and its ingredients have a wide variety of biological effects, including stimulation of gastrointestinal motility $[7,10,11,12-14]$, increases in intestinal and colonic blood flow [15-17], stimulation of motilin, vasoactive intestinal peptide, 5-hydroxytryptamine (serotonin), Calcitonin GeneRelated Peptide (CGRP) and substance P release [16,1820 ,], contraction and relaxation of intestinal smooth muscle $[21,22]$, improvement of postoperative bowel motility, ileus and adhesive obstruction [23-26], anti-inflammatory effects [27] and suppression of bacterial translocation possibly mediated by ginseng ingredients [28]. Thus, basic and clinical evidence-based studies are establishing support for the use of DKT worldwide.

In this study, we investigated the safety and the effect of DKT on body weight in infants after repair of uncomplicated gastroschisis. To our knowledge, this is the first report of the effect of DKT on gastroschisis.

\section{MATERIALS AND METHODS}

A retrospective study was performed on all infants who were diagnosed with gastroschisis and managed at our unit from June 1991 to August 2009. The surgical management and postoperative management were decided by the attending surgeons. There were five pediatric surgeons during the study period. We reviewed the infant birth history and hospital course and obtained perinatal data and clinical information from the patient records as well as from the neonatal intensive care unit database of our unit. The perinatal data included the birth date, mode of delivery, birth weight, gestational age and Apgar scores at 1 minute. Each infant's clinical history and surgical records were reviewed for intestinal atresia, necrosis, perforation, extraintestinal anomalies and mode of closure. Exclusion criteria were coexistence of other severe anomalies, gestational age of less than 32 weeks and complicated gastroschisis. The term "complicated" gastroschisis is defined by the presence of gastrointestinal defects and liver herniation, because these patients are more likely to require extensive hospital resources and to have a diminished chance of survival $[29,30]$.

Parenteral nutrition was initiated in all patients and continued until at least $80 \%$ of the caloric needs were met via enteral feeding. We treated 26 gastroschisis infants, of whom 16 infants had uncomplicated gastroschisis. Of these 16 infants, eight received $0.3 \mathrm{~g} / \mathrm{kg} /$ day of DKT (group 1: $\mathrm{n}=8$ ) and eight did not receive DKT (group 2: $\mathrm{n}=8$ ). Administration of $0.3 \mathrm{~g} / \mathrm{kg} /$ day of DKT extract powder (Tsumura \& Co., Tokyo, Japan) was pre- scribed from the first day of sustained enteral feeding for at least 12 months. If DKT administration became hard to continue after the age of 12 months owing to the complex unpleasant taste, we discontinued DKT administration with informed consent. The outcome variable was body weight as an indicator of the effect of DKT on infants with gastroschisis.

Statistical analyses were performed using StatView 4.5 (Abacus Concepts, Berkeley, CA). Clinical data were analyzed using Student's $t$ test and the Mann-Whitney U test. The differences in body weight relative to each group were estimated by the Mann-Whitney U test. Multivariate survival analyses were performed for DKT administration and variables with $\mathrm{p}$ values of less than 0.10 in Table 1. The Cox proportional hazards model was applied to test the significance of the independent influences of the clinical variables on body weight gain from birth to the ages of 9 and 12 months. Data are pre- sented as means \pm SE. Values of $\mathrm{p}<0.05$ were consi- dered to indicate statistical significance.

\section{RESULTS}

No side effects related to DKT administration were reported, such as skin eruption, abdominal pain, diarrhea and liver dysfunction. All eight infants in group 1 continued DKT administration for 12 months, although four infants $(50 \%)$ were subsequently unable to continue DKT administration because of the complex unpleasant taste of DKT after the age of 12 months. The other four infants $(50 \%)$ could continue DKT administration without side effects for our observation period.

Table 1 shows the clinical characteristics of the two groups. There were no significant differences in the background characteristics including mode of delivery and operation type. However, there were trends toward increased birth weights $(p=0.0578)$ and decreased Apgar scores $(p=0.0826)$ in group 1 compared with group 2. Although we expected that DKT administration would improve the time required to full feeds and length of hospital stay, we did not observe any significant differrences in these data (Table $\mathbf{1}$ ).

Figure 1 shows the body weight changes from 3 to 12 months of age with or without DKT administration. Although we observed an increasing trend in the birth weight of group 1 compared with group 2 (Table 1), there was no significant difference in the body weights between the two groups at the age of 3 months $(4058 \pm 331 \mathrm{~g}$ vs $3426 \pm 554 \mathrm{~g}, \mathrm{p}=0.1649)$. At the age of 6 months, we observed an increasing trend in the body weight of group 1 compared with group $2(6277 \pm 467 \mathrm{~g}$ vs $5268 \pm 398 \mathrm{~g}$, $\mathrm{p}=0.0845$ ). At the ages of 9 and 12 months, the body weights of group 1 were significantly increased compared with group 2 (9 months: $7914 \pm 539$ g vs $6612 \pm$ 
Table 1. Clinical charasteristics in the infants with and without DKT administration.

\begin{tabular}{|c|c|c|c|c|}
\hline & Group 1 & & Group 2 & $\mathrm{p}$ Value \\
\hline & & $(\mathrm{n}=8)$ & $(\mathrm{n}=8)$ & \\
\hline \multirow[t]{3}{*}{ Sex } & & & & 0.3135 \\
\hline & Male & 5 & 2 & \\
\hline & Female & 3 & 6 & \\
\hline \multirow[t]{3}{*}{$\begin{array}{c}\text { Prenatal } \\
\text { Diagnosis }\end{array}$} & & & & 0.4497 \\
\hline & $(+)$ & 8 & 6 & \\
\hline & $(-)$ & 0 & 2 & \\
\hline \multirow[t]{3}{*}{$\begin{array}{l}\text { Mode of } \\
\text { Delivery }\end{array}$} & & & & $>0.9999$ \\
\hline & Transvaginal & 2 & 2 & \\
\hline & C-Section & 6 & 6 & \\
\hline Gestational Age & (Weeks) & $36.8 \pm 0.49$ & $36.6 \pm 0.47$ & 0.5286 \\
\hline Birth Weight & (g) & $2413 \pm 169$ & $1977 \pm 80$ & 0.0578 \\
\hline Apgar Score & & $6.4 \pm 0.68$ & $7.9 \pm 0.55$ & 0.0826 \\
\hline \multirow[t]{3}{*}{ Operation } & & & & 0.2807 \\
\hline & $\begin{array}{l}\text { Primary } \\
\text { Closure }\end{array}$ & 4 & 1 & \\
\hline & $\begin{array}{l}\text { Delayed } \\
\text { Closure }\end{array}$ & 4 & 7 & \\
\hline $\begin{array}{c}\text { Time to the First } \\
\text { Feed }\end{array}$ & & $11.3 \pm 2.3$ & $14.5 \pm 5.3$ & 0.8748 \\
\hline $\begin{array}{l}\text { Time to Full } \\
\text { Feeds }\end{array}$ & (Days) & $49.7 \pm 18.7$ & $32.2 \pm 11.7$ & 0.2936 \\
\hline $\begin{array}{c}\text { Length of } \\
\text { Hospital Stay }\end{array}$ & (Days) & $77.8 \pm 18.2$ & $86.8 \pm 17.4$ & 0.5995 \\
\hline
\end{tabular}

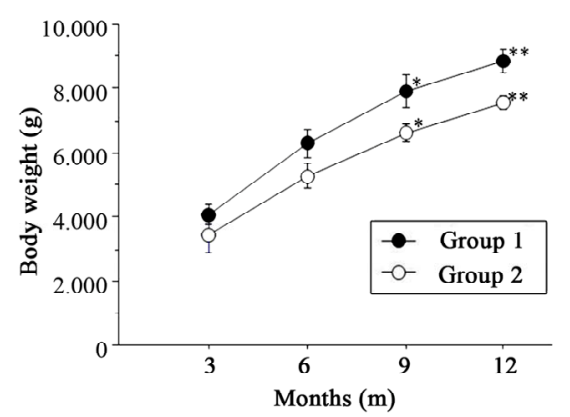

Figure 1. Body weight changes from the ages of 3 to 12 months. The infants in group 1 received $0.3 \mathrm{~g} / \mathrm{kg} /$ day of DKT, while the infants in group 2 did not receive DKT. ${ }^{*} \mathrm{p}=0.0348 ;{ }^{* * *} \mathrm{p}=0.0097$.

$278 \mathrm{~g}, \mathrm{p}=0.0348 ; 12$ months: $8290 \pm 287 \mathrm{~g}$ vs $7545 \pm$ $205 \mathrm{~g}, \mathrm{p}=0.0097$ ) (Figure 1).

We then examined the relationship between DKT administration and body weight gain at the ages of 9 and 12 months by multivariate analyses including birth weight and Apgar score, which had $\mathrm{p}$ values of less than 0.10 in Table 1. The multivariate analyses revealed that only DKT administration had a significant impact on body weight gain at the ages of 9 and 12 months ( 9 months: $p$ $=0.0201 ; 12$ months: $\mathrm{p}=0.0111$ ) (Table 2).

\section{DISCUSSION}

The reported incidence of gastroschisis ranges from 0.4 to 11.7 cases per 10,000 live births, and studies in Japan, the United States and Europe have indicated that the incidence has markedly increased in all maternal age groups over the past two decades [31-38]. Gastroschisis is a congenital condition with survival of greater than $90 \%$ [39], although most patients with gastroschisis have initial gut dysmotility and require parenteral nutrition. The etiology of the inherent dysmotility is likely to be a combination of exposure to amniotic fluid and constriction of the bowel at the abdominal wall defect, and much of the damage seems to occur toward the end of pregnancy [40-44], although there is currently no evidence that amniotic exchange improves postnatal bowel motility in humans. Delayed maturation of the intestinal pacemaker and smooth muscle cells has been demonstrated in a rat model of gastroschisis [45], which may explain the delayed onset of peristalsis in these patients. However, its etiology has not yet been completely unveiled, and there is no safe and effective treatment for the intestinal dysmotility other than awaiting spontaneous resolution [2, $3,46]$.

The main ingredients of DKT include hydroxy-asanshool (Japanese pepper), 6-shogaol (processedginger), and ginsenoside $\mathrm{Rb} 1$ (ginseng radix). Contamination studies have certified DKT to be free of unexpected pharmaceutical ingredients, toxins, pesticides, microbes, and heavy metals [7].Several important, neurally mediated mechanisms have been suggested as mediating the increased effective intestinal motility of DKT [12,47].One of these mechanisms of action involves there lease of CGRP, a neuropeptide produced by the sensory neurons of the gut. Calcitonin gene-related peptide is one of the most potent mediators of microvascular vasodilation in the human body and is also known for its secretory, antiinflammatory, and trophic actions in the gut. As a protein with broad spectrum of functions, the role of CGRP in gut function may illuminate key pathways for understanding the diverseactions of DKT including improvement of gastrointestinal motility and intestinal blood flow and even in the prevention of intestinal adhesions resulting from inflammation [7].

In this retrospective study, we investigated the effect of DKT administration on body weight gain in infants with uncomplicated gastroschisis. We administered DKT at a dose of $0.3 \mathrm{~g} / \mathrm{kg} /$ day from the first day of sustained 
Table 2. Multiple linear regression evaluating the effect of DKT administration, birth weight and Apgar score on body weight gain at the age of 9 months and 12 months.

\begin{tabular}{cccccccc}
\hline \multicolumn{3}{c}{ Body Weight Gain at the Age of 9 Months } & \multicolumn{3}{c}{ Body Weight Gain at the Age of 12 Months } \\
\hline \multicolumn{2}{c}{ Variables } & {$[\beta]$} & $95 \%$ CI & p & {$[\beta]$} & $95 \%$ CI & p \\
DKT Administration (yes $=1)$ & 2.828 & $(0.465$ to 5.350$)$ & 0.0201 & 3.994 & $(0.901$ to 7.872$)$ & 0.0111 \\
Birth Weight (g) & 0.001 & $(-0.001$ to 0.004$)$ & 0.3933 & 0.002 & $(-0.001$ to 0.006$)$ & 0.1596 \\
Apgar Score & -0.519 & $(-1.104$ to 0.027$)$ & 0.0618 & -0.625 & $(-1.619$ to 0.108$)$ & 0.0981 \\
\hline
\end{tabular}

enteral feeding to at least 12 months of age. We observed that the body weight of the DKT administration group was significantly increased compared with that of the non-administration group at the ages of 9 and 12 months. Furthermore, multivariate analyses revealed that only DKT administration had a significant impact on the body weight gain at the ages of 9 and 12 months. We consider that these observations originated from the pharmacological effect of DKT on the intestinal dysmotility in the infants with gastroschisis. We further suggest that DKT administration may be safe in infants, because DKT is composed of natural herbs and no side effects related to DKT administration were reported.

We did not observe any significant differences in the data for the time to full feeds and length of hospital stay. Two possibilities may account for these observations. First, the dose of $0.3 \mathrm{mg} / \mathrm{kg} /$ day may be a low dose for infants, and we may need to increase the dose to 0.6 $\mathrm{mg} / \mathrm{kg} /$ day. Second, the start of the DKT administration may be late, since we started it from the first day of sustained enteral feeding (group 1:11.3 days; group 2:14.5 days). Therefore, we may need to start administration from the early postoperative phase using a nasogastric tube before sustained enteral feeding.

The problem associated with DKT is its complex unpleasant taste (predominantly bitter or astringent) owing to several of its active constituents, which frequently causes noncompliance. Four infants $(50 \%)$ were unable to continue DKT administration because of the complex unpleasant taste of DKT after the age of 12 months in our study. We therefore suggest that DKT needs to be tastemasked to improve compliance, especially for children.

Our study has several limitations. First, the sample size was small because it was a single-center retrospective study. Second, we did not analyze the laboratory data, including rapid turn-over protein, gastric emptying and intestinal transit time. Third, the surgical management and postoperative management were decided by five attending surgeons. These points should be analyzed in future prospective studies.

In conclusion, this study has shown that DKT can increase the body weight gain in postoperative infants with gastroschisis. DKT administration might work safely and effectively for improving the body weight gain in infants with uncomplicated gastroschisis. Further studies with larger samples or randomized controlled trials are necessary to obtain more accurate data for this medicine.

\section{REFERENCES}

[1] Christison-Lagay, E.R., Kelleher, C.M. and Langer, J.C. (2011) Neonatal abdominal wall defects. Seminars in Fetal and Neonatal Medicine, 16, 164-172.

[2] Dicken, B.J., Sergi, C., Rescorla, F.J., Breckler, F., et al. (2011) Medical management of motility disorders in patients with intestinal failure: A focus on necrotizing enterocolitis, gastroschisis, and intestinal atresia. Journal of Pediatric Surgery, 46, 1618-1630.

[3] Curry, J.I., Lander, A.D. and Stringer, M.D. (2004) A multicenter, randomized, double-blind, placebo-controlled trial of the prokinetic agent erythromycin in the postoperative recovery of infants with gastroschisis. Journal of Pediatric Surgery, 39, 565-569.

[4] Lander, A., Redkar, R., Nicholls, G., Lawson, A., et al. (1997) Cisapride reduces neonatal postoperative ileus: Randomised placebo controlled trial. Archives of Disease in Childhood. Fetal and Neonatal, 77, 119-122. doi:10.1136/fn.77.2.F119

[5] Itoh, T., Yamakawa, J., Mai, M., Yamaguchi, N., et al. (2002) The effect of the herbal medicine Dai-kenchu-to on post-operative ileus. The Journal of International Medical Research, 30, 428-432.

[6] Ohya, T., Usui, Y., Arii, S., Iwai, T. et al. (2003) Effect of Dai-kenchu-to on obstructive bowel disease in children. The American Journal of Chinese Medicine, 31, 129-135.

[7] Kono, T., Kanematsu, T. and Kitajima, M. (2009) Exodus of Kampo, traditional Japanese medicine, from the complementary and alternative medicines: Is it time yet? Surgery, 146, 837-840.

[8] Manabe, N., Camilleri, M., Rao, A., Wong, B.S., et al. (2010) Effect of Dai-kenchu-to (TU-100) on gastro-intesti- nal and colonic transit in humans. American Journal of Physiology-Gastrointestinal and Liver Physiology, 298, 970-975.

[9] Iwai, N., Kume, Y., Kimura, O., Ono, S., et al. (2007) Effects of herbal medicine Dai-kenchu-to on anorectal function in children with severe constipation. European Journal of Pediatric Surgery, 17, 115-118. doi:10.1055/s-2007-965016 
[10] Fukuda, H., Chen, C., Mantyh, C., Ludwig, K., et al. (2006) The herbal medicine, Dai-kenchu-to, accelerates delayed gastrointestinal transit after the operation in rats. Journal of Surgical Research, 131, 290-295.

[11] Mochiki, E., Yanai, M., Ohno, T. and Kuwano, H. (2010) The effect of traditional Japanese medicine (Kampo) on gastrointestinal function. Surgery Today, 40, 1105-1111. doi:10.1007/s00595-010-4388-8

[12] Shibata, C., Sasaki, I., Naito, H., Ueno, T., et al. (1999) The herbal medicine Dai-kenchu-to stimulates upper gut motility through cholinergic and 5-hydroxytryptamine 3 receptors in conscious dogs. Surgery, 126, 918-924.

[13] Satoh, K., Kase, Y., Hayakawa, T., Murata, P., et al. (2001) Dai-kenchu-to enhances accelerated small intestinal movement. Biological \& Pharmaceutical Bulletin, 24, 1122-1126. doi:10.1248/bpb.24.1122

[14] Kawasaki, N., Nakada, K., Nakayoshi, T., Furukawa, Y., et al. (2007) Effect of Dai-kenchu-to on gastrointestinal motility based on differences in the site and timing of administration. Digestive, Diseases, Sciences, 52, 26842694. doi:10.1007/s10620-006-9391-y

[15] Murata, P., Kase, Y., Ishige, A., Sasaki, H., et al. (2002) The herbal medicine Dai-kenchu-to and one of its active components 6-Shogaol increase intestinal blood flow in rats. Life Sciences, 70, 2061-2070. doi:10.1016/S0024-3205(01)01552-1

[16] Kono, T., Koseki, T., Chiba, S., Ebisawa, Y., et al. (2008) Colonic vascular conductance increased by Dai-kenchu-to via calcitonin gene-related peptide and receptor-activity modifying protein 1. Journal of Surgical Research, 150, 78-84.

[17] Kono, T., Omiya, Y., Hira, Y., Kaneko, A., et al. (2011) Daikenchuto (TU-100) ameliorates colon microvascular dysfunction via endogenous adrenomedullin in Crohn's disease rat model. Journal of Gastroenterology, 46, 11871196. doi:10.1007/s00535-011-0438-2

[18] Nagano, T., Itoh, H. and Takeyama, M. (1999) Effect of Dai-kenchu-to on levels of 3 brain-gut peptides (motilin, gastrin and somatostatin) in human plasma. Biological and Pharmaceutical Bulletin, 22, 1131-1133. doi:10.1248/bpb.22.1131

[19] Sato, Y., Inoue, S., Katagiri, F., Itoh, H., et al. (2006) Effects of pirenzepine on Dai-kenchu-to-induced elevation of the plasma neuropeptide levels in humans. Biological and Pharmaceutical Bulletin, 29, 166-171.

[20] Nagano, T., Itoh, H. and Takeyama, M. (2000) Effects of Dai-kenchu-to on levels of 5-hydroxytryptamine (serotonin) and vasoactive intestinal peptides in human plasma. Biological and Pharmaceutical Bulletin, 23, 352-353. doi:10.1248/bpb.23.352

[21] Kito, Y. and Suzuki, H. (2006) Effects of Dai-kenchu-to on spontaneous activity in the mouse small intestine. Journal of Smooth Muscle Research, 42, 189-201.

[22] Akiho, H. and Nakamura, K. (2011) Dai-kenchu-to ameliorates muscle hypercontractility in a murine T-cell-mediated persistent gut motor dysfunction model. Digestion, 83, 173-179.

[23] Satoh, K., Hayakawa, T., Kase, Y., Ishige, A., et al. (2001)
Mechanisms for contractile effect of Dai-kenchu-to in isolated guinea pig ileum. Digestive, Diseases, Sciences, 46, 250-256. doi:10.1023/A:1005636412287

[24] Yasunaga, H., Miyata, H., Horiguchi, H., Kuwabara, K., et al. (2011) Effect of the Japanese herbal kampo medicine Dai-kenchu-to on postoperative adhesive small bowel obstruction requiring long-tube decompression: A propensity score analysis. Evidence-Based Complementary and Alternative Medicine, 2011, 264-289. doi: $10.1155 / 2011 / 264289$

[25] Tokita, Y., Yuzurihara, M., Sakaguchi, M., Satoh, K., et al. (2007) The pharmacological effects of Dai-kenchu-to, a traditional herbal medicine, on delayed gastrointestinal transit in rat postoperative ileus. Journal of Pharmacological Sciences, 104, 303-310.

[26] Tokita, Y., Yamamoto, M., Satoh, K., Nishiyama, M., et al. (2011) Possible involvement of the transient receptor potential vanilloid type 1 channel in postoperative adhesive obstruction and its prevention by a kampo (traditional Japanese) medicine, Dai-kenchu-to. Journal of Pharmacological Sciences, 115, 75-83.

[27] Kono, T., Kaneko, A., Hira, Y., Suzuki, T. et al. (2010) Anticolitis and adhesion effects of daikenchuto via endogenous adrenomedullin enhancement in Crohn's disease mouse model. Journal of Crohn's and Colitis, 4, 161170.

[28] Yoshikawa, K., Kurita, N., Higashijima, J., Miyatani, T., et al. (2008) Kampo medicine "Dai-kenchu-to" prevents bacterial translocation in rats. Digestive, Diseases, Sciences, 53, 1824-1831. doi:10.1007/s10620-008-0281-3

[29] Molik, K.A., Gingalewski, C.A., West, K.W., Rescorla, F.J., et al. (2001) Gastroschisis: A plea for risk categorization. Journal of Pediatric Surgery, 36, 51-55.

[30] Abdullah, F., Arnold, M.A., Nabaweesi, R., Fischer, A.C., et al. (2007) Gastroschisis in the United States 1988-2003: Analysis and risk categorization of 4344 patients. Journal of Perinatology, 27, 50-55.

[31] Baird, P.A. and MacDonald, E.C. (1981) An epidemiologic study of congenital malformations of the anterior abdominal wall in more than half a million consecutive live births. The American Journal of Human Genetics, 33, 470-478.

[32] Wilson, R.D. and Johnson, M.P. (2004) Congenital abdominal wall defects: An update. Fetal Diagnosis and Therapy, 19, 385-398.

[33] Suita, S., Okamatsu, T., Yamamoto, T., Handa, N. et al. (2000) Changing profile of abdominal wall defects in Japan: Results of a national survey. Journal of Pediatric Surgery, 35, 66-71.

[34] Eggink, B.H., Richardson, C.J., Malloy, M.H. and Angel, C.A. (2006) Outcome of gastroschisis: A 20-year case review of infants with gastroschisis born in Galveston, Texas. Journal of Pediatric Surgery, 41, 1103-1108.

[35] Rasmussen, S.A. and Frias, J.L. (2008) Non-genetic risk factors for gastroschisis. American Journal of Medical Genetics, 148, 199-212. doi:10.1002/ajmg.c.30175

[36] Castilla, E.E., Mastroiacovo, P. and Orioli, I.M. (2008) Gastroschisis: International epidemiology and public health 
perspectives. American Journal of Medical Genetics, 148, 162-179. doi:10.1002/ajmg.c.30181

[37] Loane, M., Dolk, H. and Bradbury, I. (2007) Increasing prevalence of gastroschisis in Europe 1980-2002: A phenomenon restricted to younger mothers. Paediatric and Perinatal Epidemiology, 21, 363-369.

[38] Alvarez, S.M. and Burd, R.S. (2007) Increasing prevalence of gastroschisis repairs in the United States: 19962003. Journal of Pediatric Surgery, 42, 943-946.

[39] Kitchanan, S., Patole, S.K., Muller, R. and Whitehall, J.S. (2000) Neonatal outcome of gastroschisis and exomphalos: A 10-year review. Journal of Paediatrics and Child Health, 36, 428-430. doi:10.1046/j.1440-1754.2000.00551.x

[40] Curry, J.I., McKinney, P., Thornton, J.G. and Stringer, M.D. (2000) The aetiology of gastroschisis. BJOG: An International Journal of Obstetrics and Gynaecology, 107, 1339-1346. doi:10.1111/j.1471-0528.2000.tb11645.x

[41] Dilsiz, A., Gundogan, A.H., Aktan, M., Duman, S. et al. (1999) Nitric oxide synthase inhibition prevents intestinal damage in gastroschisis: A morphological evaluation in chick embryos. Journal of Pediatric Surgery, 34, 12481252.

[42] Fasching, G., Haeusler, M., Mayr, J., Schimpl, G. et al. (2005) Can levels of interleukins and matrix metallopro- teinases in the amniotic fluid predict postnatal bowel function in fetuses with gastroschisis. Journal of Pediatric Surgery, 40, 1887-1891.

[43] Langer, J.C., Longaker, M.T., Crombleholme, T.M., Bond, S.J., et al. (1989) Etiology of intestinal damage in gastroschisis. I: Effects of amniotic fluid exposure and bowel constriction in a fetal lamb model. Journal of Pediatric Surgery, 24, 992-997.

[44] Langer, J.C., Bell, J.G., Castillo, R.O., Crombleholme, T.M., et al. (1990) Etiology of intestinal damage in gastroschisis, II. Timing and reversibility of histological changes, mucosal function, and contractility. Journal of Pediatric Surgery, 25, 1122-1126.

[45] Midrio, P., Faussone-Pellegrini, M.S., Vannucchi, M.G. and Flake, A.W. (2004) Gastroschisis in the rat model is associated with a delayed maturation of intestinal pacemaker cells and smooth muscle cells. Journal of Pediatric Surgery, 39, 1541-1547.

[46] Keys, C., Drewett, M. and Burge, D.M. (2008) Gastroschisis: The cost of an epidemic. Journal of Pediatric Surgery, 43, 654-657.

[47] Sato, Y., Katagiri, F., Inoue, S., Itoh, H. et al. (2004) Daikenchu-to raises levels of calcitonin gene-related peptide and substance $\mathrm{P}$ in human plasma. Biological and Pharmaceutical Bulletin, 27, 1875-1877. 